ARTICLE

Received 10 Sep 2012 | Accepted 8 Mar 2013 | Published 16 Apr $2013 \quad$ DOl: 10.1038/ncomms2722

\title{
Highly sensitive sulphide mapping in live cells by kinetic spectral analysis of single Au-Ag core-shell nanoparticles
}

Bin Xiong ${ }^{1}$, Rui Zhou ${ }^{1}$, Jinrui Hao ${ }^{1}$, Yanghui Jia', Yan He${ }^{1} \&$ Edward S. Yeung ${ }^{1}$

Hydrogen sulphide $\left(\mathrm{H}_{2} \mathrm{~S}\right)$ is a gaseous signalling agent that has important regulatory roles in many biological systems but remains difficult to measure in living biological specimens. Here we introduce a new method for highly sensitive sulphide mapping in live cells via singleparticle plasmonic spectral imaging that uses Au-Ag core-shell nanoparticles as probes. This strategy is based on $\mathrm{Ag}_{2} \mathrm{~S}$ formation-induced spectral shifts of the nanoprobes, which is not only highly selective towards sulphide but also shows a linear logarithmic dependence on sulphide concentrations from $0.01 \mathrm{nM}$ to $10 \mu \mathrm{M}$. A theoretical model was established that successfully explained the experimental observations, suggesting that the local sulphide concentration as well as its oscillations can be determined indirectly from kinetic measurements of the spectral shifts of the nanoprobes. We demonstrated for the first time the realtime mapping of local variations of sulphide levels in live cells with nM sensitivity.

\footnotetext{
${ }^{1}$ State Key Laboratory of Chemo/Biosensing and Chemometrics, College of Chemistry and Chemical Engineering, College of Biology, Hunan University, Changsha 410082, China. Correspondence and requests for materials should be addressed to Y.H. (email: yanhe2021@gmail.com).
} 
ydrogen sulphide $\left(\mathrm{H}_{2} \mathrm{~S}\right)$, traditionally considered a toxic gas with a rotten egg smell, has been recognized as an endogenous gasotransmitter with importance comparable to nitric oxide (NO) and carbon monoxide (CO), and has attracted increasing attention in recent years ${ }^{1-4} . \mathrm{H}_{2} \mathrm{~S}$ equilibrates with aqueous sulphides ( $\mathrm{HS}^{-}$and $\mathrm{S}^{2-}$ ) in biological environments and has vital roles in various physiological and pathological processes, including vasorelaxation ${ }^{5}$, angiogenesis ${ }^{6}$, cerebral microcirculation $^{7}$, neurotransmission ${ }^{8,9}$, inflammation ${ }^{10}$, apoptosis $^{11}$, insulin signalling inhibition ${ }^{12}$, oxygen sensing ${ }^{13}$ and energy production in mitochondria ${ }^{14}$. Meanwhile, the $\mathrm{H}_{2} \mathrm{~S}$ levels are related to a number of diseases, such as Down syndrome ${ }^{15}$, Alzheimer disease $^{16}$, hyperglycaemia ${ }^{17}$, ischaemia ${ }^{18}$ and so on. To elucidate the complex behaviours of $\mathrm{H}_{2} \mathrm{~S}$ in both healthy and disease states, it is crucial to measure $\mathrm{H}_{2} \mathrm{~S}$ distribution in biological systems in real time. Indeed, conventional assays using colorimetric analysis ${ }^{19}$, electrochemical analysis ${ }^{20,21}$ and gas chromatography ${ }^{22}$ are currently available to determine $\mathrm{H}_{2} \mathrm{~S}$ concentrations in processed samples, such as homogenized tissues, cell lysates or blood serum. During the past several years, several research groups have developed sulphide-responsive fluorescent organic molecules to detect $\mathrm{H}_{2} \mathrm{~S}$ in live cells and have achieved selective intracellular sulphide imaging with limits of detection of $1-10 \mu \mathrm{M}$ (ref. 23-28). Although most publications suggest that the average endogenous $\mathrm{H}_{2} \mathrm{~S}$ level is in the $\mu \mathrm{M}$ range, much lower sulphide concentrations have been reported ${ }^{29}$. More importantly, the anabolism and catabolism of cellular sulphide are known to be rapid, which means that the sulphide concentration could fluctuate continuously from as high as sub$\mathrm{mM}$ during its explosive production to as low as a few $\mathrm{nM}$ after its rapid consumption ${ }^{30}$. However, almost all existing $\mathrm{H}_{2} \mathrm{~S}$ sensing methods are based on some irreversible stoichiometric reaction between the probe and sulphide species ${ }^{23-28}$ and cannot be utilized to monitor the dynamics of sulphide generation and elimination inside cells, especially at the single-molecule level. Therefore, more sensitive methods that are capable of following time-dependent changes in $\mathrm{H}_{2} \mathrm{~S}$ concentrations over a large dynamic range are needed for in-depth studies of this gaseous transmitter.

Plasmonic nanoparticles (PNPs) have long been utilized for chemical and biological sensing ${ }^{31}$, owing to their unique size, shape and composition as well as local environment-dependent optical properties ${ }^{32,33}$. Compared with fluorescent organic molecules, PNPs are very bright, photostable and easy to prepare. The scattering cross-section of a $54 \times 14-\mathrm{nm}$ gold nanorod (AuNR) at its localized surface plasmon resonance (LSPR) wavelength, for example, is $\sim 10^{5}$ times that of a fluorophore, making large single PNPs and their spectral changes readily observable with dark-field microscopy ${ }^{34,35}$. In addition to high sensitivity, like other single-molecule spectroscopy techniques, single PNP probes and imaging platforms provide much improved spatiotemporal resolution compared with bulk measurements. These all facilitate in situ investigations of biological processes and localized chemical reactions ${ }^{36,37}$.

In this study, we present an indirect, 'integrative' approach for kinetic mapping of sulphide in live cells by using single-particle spectral imaging with single gold nanorod-silver (AuNR-Ag) core-shell PNPs as probes. In this plasmonic nanoprobe, the Ag shell acts as the sensing agent that reacts with the sulphide, and the AuNR core has the role of the signal reporter, whose spectrum shifts to longer wavelengths when $\mathrm{Ag}_{2} \mathrm{~S}$ forms. Although the $\mathrm{Ag}_{2} \mathrm{~S}$ formation reaction is irreversible, as is the case with most reported $\mathrm{H}_{2} \mathrm{~S}$-sensing reactions, the present study demonstrates both experimentally and theoretically that the reaction rate, and therefore, the rate of the induced spectral shifts of the PNP probes are correlated with the surrounding sulphide concentration. As a result, local intracellular sulphide concentrations and their variations were able to be determined in real time with nM sensitivity over a dynamic range of six orders of magnitude. To our knowledge, this is the first time that an intracellular gaseous transmitter has been monitored using single PNPs as optical probes.

\section{Results}

Sulphide sensing in bulk solution. It is well known that in the presence of oxygen, sulphide ions selectively and quickly react with $\mathrm{Ag}$ atoms to generate $\mathrm{Ag}_{2} \mathrm{~S}$ at room temperature ${ }^{38,39}$,

$4 \mathrm{Ag}+2 \mathrm{~S}^{2-}+\mathrm{O}_{2}+2 \mathrm{H}_{2} \mathrm{O} \rightarrow 2 \mathrm{Ag}_{2} \mathrm{~S}+4 \mathrm{OH}^{-}$

Because the refractive indices of $\mathrm{Ag}(\sim 0.17)$ and $\mathrm{Ag}_{2} \mathrm{~S}(\sim 2.2)$ are substantially different ${ }^{40,41}$, the formation of $\mathrm{Ag}_{2} \mathrm{~S}$ on an $\mathrm{Au}-\mathrm{Ag}$ core-shell nanoparticle surface would result in its LSPR maximum shifting to a longer wavelength. AuNR is chosen as the core material because, as reported in the literature ${ }^{34,42}$, AuNRs with large aspect ratios, and thus LSPR maxima at long wavelengths, are highly sensitive to local refractive index variations. Accordingly, this AuNR-Ag nanoprobe exhibits a detectable colour change or redshift in its extinction spectrum even in the presence of a miniscule amount of sulphide, an advantage that could be utilized for sensitive and selective sulphide sensing.

Figure 1 shows the bulk performance of AuNR-Ag PNPs having a $74 \times 19-\mathrm{nm}$ AuNR core and a 2.1-nm Ag shell. The longitudinal peak $\left(\lambda_{\max }\right)$ of their LSPR spectrum occurs at $779 \mathrm{~nm}$ originally but shifts to longer wavelengths after addition of $\mathrm{Na}_{2} \mathrm{~S}$ (as an aqueous $\mathrm{H}_{2} \mathrm{~S}$ source). The $\lambda_{\max }$ change $\left(\Delta \lambda_{\max }\right)$ of the PNP solution $(\sim 50 \mathrm{pM})$ increases with $\mathrm{Na}_{2} \mathrm{~S}$ concentration, and a limit of detection of $0.1 \mu \mathrm{M}$ is achieved. The time courses of $\Delta \lambda_{\max }$ after adding either $5 \mathrm{mM}$ or $5 \mu \mathrm{M} \mathrm{Na} \mathrm{N}_{2} \mathrm{~S}$ to the PNP solution indicate that the PNP responds rapidly to both low and high sulphide concentrations. Because the concentrations of biothiols, such as cysteine and glutathione, in biological specimens are at $\mathrm{mM}$ levels ${ }^{43,44}$, the selectivity of the sulphide nanoprobe in the presence of glutathione and cysteine was tested. The spectral shifts of the PNPs induced by $5 \mathrm{mM}$ of either biothiol are only $\sim 2 \mathrm{~nm}$, which is negligible compared with that induced by $5 \mu \mathrm{M}$ sulphide. We also examined the responses of the nanoprobes towards inorganic sulphur ions $\left(\mathrm{S}_{2} \mathrm{O}_{3}^{2-}, \mathrm{SO}_{3}^{2-}\right.$, $\left.\mathrm{SCN}{ }^{-}\right)$and other anions $\left(\mathrm{NO}_{3}^{-}, \mathrm{NO}_{2}^{-}, \mathrm{Cl}^{-}, \mathrm{Br}^{-}, \mathrm{I}^{-}\right.$, $\left.\mathrm{CH}_{3} \mathrm{COO}^{-}, \mathrm{PO}_{4}^{3-}, \mathrm{CO}_{3}^{2-}\right)$. Because the solubility of $\mathrm{Ag}_{2} \mathrm{~S}$ $(\mathrm{pKsp}=50.83)$ is much lower than other Ag compounds, no comparable LSPR responses from other species were detected even though their concentrations were much higher.

Sulphide detection via single-particle spectral imaging. Although the PNP probes have performed as well as the fluorescent probes reported previously ${ }^{23-28}$, due to the ensemble average effect, bulk measurements are intrinsically not as sensitive as single-particle measurements. The AuNR-Ag PNP with a 2.1-nm thick silver shell, for example, contains $\sim 6 \times 10^{5}$ silver atoms, which translates to $\sim 30 \mu \mathrm{M}$ Ag for a $\sim 50 \mathrm{pM}$ PNP solution. Thus, if the target sulphide concentration in the PNP solution is too low (for example, $<0.1 \mu \mathrm{M}$ ), the total amount of $\mathrm{Ag}_{2} \mathrm{~S}$ formation would not be enough to induce appreciable $\Delta \lambda_{\max }$ shifts for all PNPs. However, if the concentration of PNPs is reduced too much to allow more fractional coverage of $\mathrm{Ag}_{2} \mathrm{~S}$ on each PNP, the overall optical response will not have a large enough signal-to-noise-ratio to be detected by ultraviolet-vis spectrometers. This dilemma can be resolved by monitoring the spectral shifts of individual PNPs separately using single-particle spectroscopy. Because each AuNR-Ag NP functions as an 

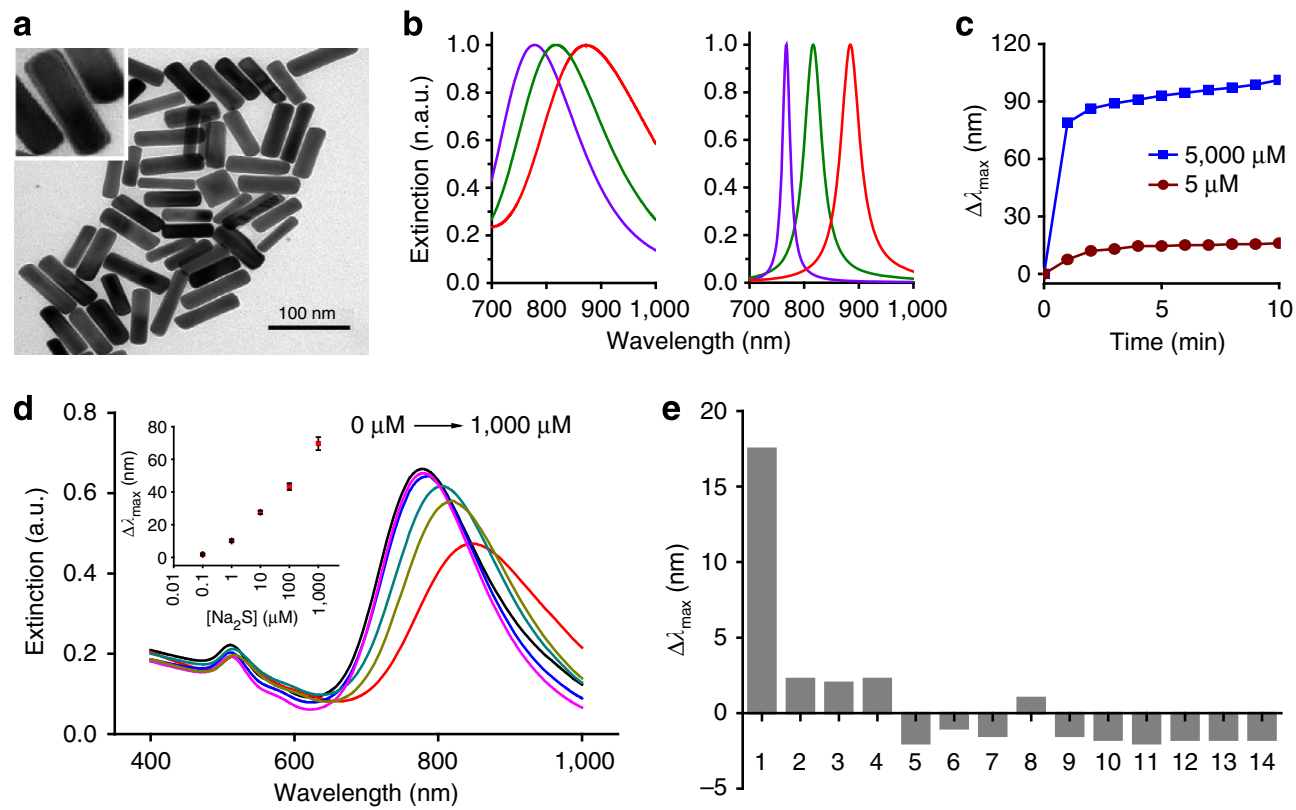

Figure 1 | Sulphide sensing in bulk solution with AuNR-Ag core-shell nanoprobes. (a) TEM characterization of AuNR-Ag nanoprobes with a $74 \times 19-n m$ AuNR core and a 2.1-nm Ag shell. (b) Experimental (left) and DDA-simulated (right) LSPR spectra of AuNR (green), AuNR-Ag (purple) and AuNR-Ag $2 \mathrm{~S}$ nanoparticles. (c) Extinction spectra and $\Delta \lambda_{\max }$ shifts (insert) of $\sim 50 \mathrm{pM}$ PNP solution immediately after adding $0.1,1,10,100$ and $1000 \mu \mathrm{M}$ of $\mathrm{Na}_{2} \mathrm{~S}$. (d) Time courses of $\Delta \lambda_{\max }$ increase with the addition of $5 \mu \mathrm{M}$ and $5 \mathrm{mM} \mathrm{Na} 2 \mathrm{~S}$. (e) Selectivity for biothiols, inorganic sulphur ions and other anions. Their concentration is $25 \mu \mathrm{M}$ unless indicated otherwise. Legend: $1, \mathrm{Na}_{2} \mathrm{~S}(5 \mu \mathrm{M}) ; 2$, cysteine ( $\left.5 \mathrm{mM}\right) ; 3$, glutathione $\left(5 \mathrm{mM}^{2} ; 4, \mathrm{Na}_{2} \mathrm{~S}_{2} \mathrm{O}_{3} ; 5, \mathrm{Na}_{2} \mathrm{SO}_{3} ; 6, \mathrm{KSCN} ; 7\right.$ $\mathrm{NaCl} ; 8, \mathrm{NaBr} ; 9, \mathrm{Nal} ; 10, \mathrm{NaNO}_{2} ; 11 . \mathrm{NaNO}_{3} ; 12, \mathrm{CH}_{3} \mathrm{COONa} ; 13, \mathrm{Na}_{3} \mathrm{PO}_{4} ; 14, \mathrm{Na}_{2} \mathrm{CO}_{3}$.

independent sulphide probe, no minimum concentration of PNPs is required. The effective 'detection volume' of every nanoprobe is, thus, enlarged, making it possible to accumulate or react with more sulphides locally to achieve a lower detection limit. The imaging method utilized in the present study, which was previously developed in our laboratory, is a high-throughput single-particle dark-field spectral imaging technique ${ }^{45}$, in which a transmission grating beam splitter (TGBS) is placed in the light collection path (Supplementary Fig. S1). The TGBS splits the scattering light emitted from each PNP into a zero-order image of a focused spot and a first-order image of a long streak, and the peak-to-peak distance (after Gaussian fitting) between the spot and the streak is proportional to $\lambda_{\max }$ of the particle, as dictated by Bragg diffraction. Compared with a commercial spectrograph equipped with an entrance slit, this slit-less technique can follow the spectral shifts of multiple particles or even moving particles simultaneously. To match the optimal spectral response window of the CCD camera, we prepared AuNR-Ag NPs with a 3-nm Ag shell $\left(\lambda_{\max }=622 \mathrm{~nm}\right.$, Supplementary Fig. S2) as the single PNP probe used in all single-particle experiments.

Figure 2a,b display typical single-particle spectral images before and after adding $\mathrm{Na}_{2} \mathrm{~S}$, respectively. The separations between the first-order images and the corresponding zero-order images clearly increase with $\mathrm{Ag}_{2} \mathrm{~S}$ formation. To investigate their sensitivity and dynamic range, we immersed single PNP probes, which were immobilized on a glass substrate, in $\mathrm{Na}_{2} \mathrm{~S}$ solutions of different concentrations for $30 \mathrm{~min}$ and recorded their resulting spectral images. By Gaussian fitting the first-order streaks from randomly selected nanoprobes at each $\mathrm{Na}_{2} \mathrm{~S}$ concentration, a Gaussian-shaped distribution of $\lambda_{\max }$ values was obtained (Fig. 2c). All distributions were $\sim 30 \mathrm{~nm}$ in width and appeared uncorrelated with $\mathrm{Ag}_{2} \mathrm{~S}$ formation, suggesting that the difference in $\lambda_{\max }$ values at the same concentration can be attributed to variations in the aspect ratios and silver shell thicknesses of single nanorods. We note that these single-particle experiments were performed with $\mathrm{Na}_{2} \mathrm{~S}$ water solution, but due to the high selectivity of the nanoprobes, the results were nearly the same when $150 \mathrm{mM} \mathrm{NaCl}$ and $5 \mathrm{mM}$ cysteine or glutathione were also present (Supplementary Fig. S3). The relationship between the mean values of $\lambda_{\max }$ distributions and $\mathrm{Na}_{2} \mathrm{~S}$ concentrations is plotted in Figure $2 \mathrm{~d}$. An $\sim 2$-nm shift in the $\lambda_{\max }$ distribution is observed when the single NP probes are exposed to a solution of just $0.01 \mathrm{nM} \mathrm{Na} 2 \mathrm{~S}$. Amazingly, the mean $\Delta \lambda_{\max }$ value apparently scales up linearly with the logarithm of the applied sulphide concentration from $0.01 \mathrm{nM}$ to $10 \mu \mathrm{M}$ for six orders of magnitude. As far as we know, such a large dynamic range has never been reported for spectrally responsive single-molecule or single-particle probes.

Sulphide concentration determination from PNP spectral shift rates. To understand the underlying mechanism, we set out to study the relationship between $\Delta \lambda_{\max }$ and $C_{\mathrm{S}, \mathrm{NP}}$, the sulphide concentration one AuNR-Ag core-shell PNP consumes to produce $\mathrm{Ag}_{2} \mathrm{~S}$ on its surface. From theoretical derivations (Supplementary Note 1 ), we can tell that $\Delta \lambda_{\max }$ is proportional to the local refractive index variation, $\Delta n$ (ref. 32,46), which is further proportional to the molar fraction of $\mathrm{Ag}_{2} \mathrm{~S}$ formed in the silver shell, $f=C_{\mathrm{S}, \mathrm{NP}} /\left(C_{\mathrm{Ag}, 0}-C_{\mathrm{S}, \mathrm{NP}}\right)$, where $C_{\mathrm{Ag}, 0}$ is the total concentration of $\mathrm{Ag}$ atoms available. Supplementary Figure S4 plots the calculated $\Delta \lambda_{\max }$ as a function of $C_{\mathrm{S}, \mathrm{NP}}$. It can be seen that while the dependence is not linear, it is by no means a logarithmic relationship. This is reasonable because $C_{\mathrm{Ag}, 0}$ is a constant, and the maximum $C_{\mathrm{S}, \mathrm{NP}}$ value is limited regardless of the external sulphide concentration, $C_{\mathrm{S}, \mathrm{NP}}=C_{\mathrm{Ag}_{2} \mathrm{~S}} \leq C_{\mathrm{Ag}, 0} / 2$. Therefore, there must be a kinetic equilibrium between the external $\mathrm{Na}_{2} \mathrm{~S}$ concentration applied, $C_{\mathrm{S}, 0}$, and the effective concentration of sulphide capable of reacting with the silver shell, 
a

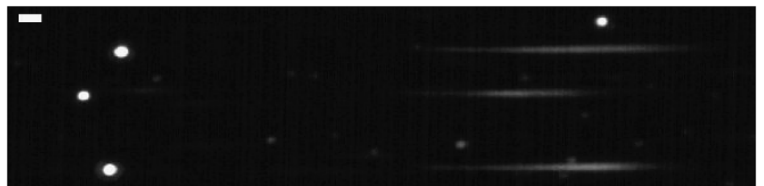

b
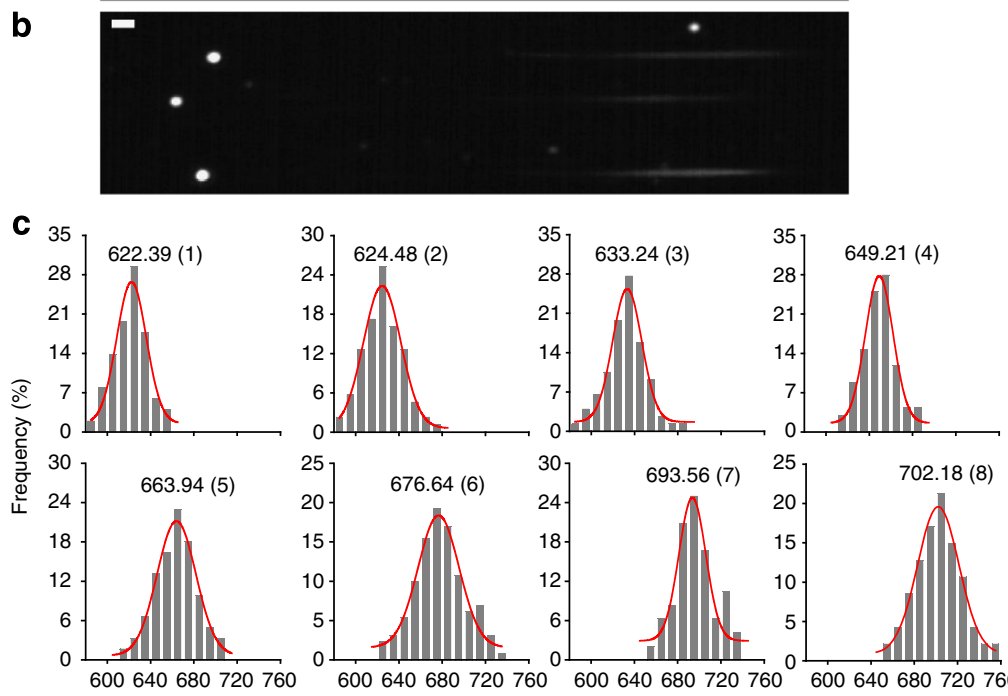

d
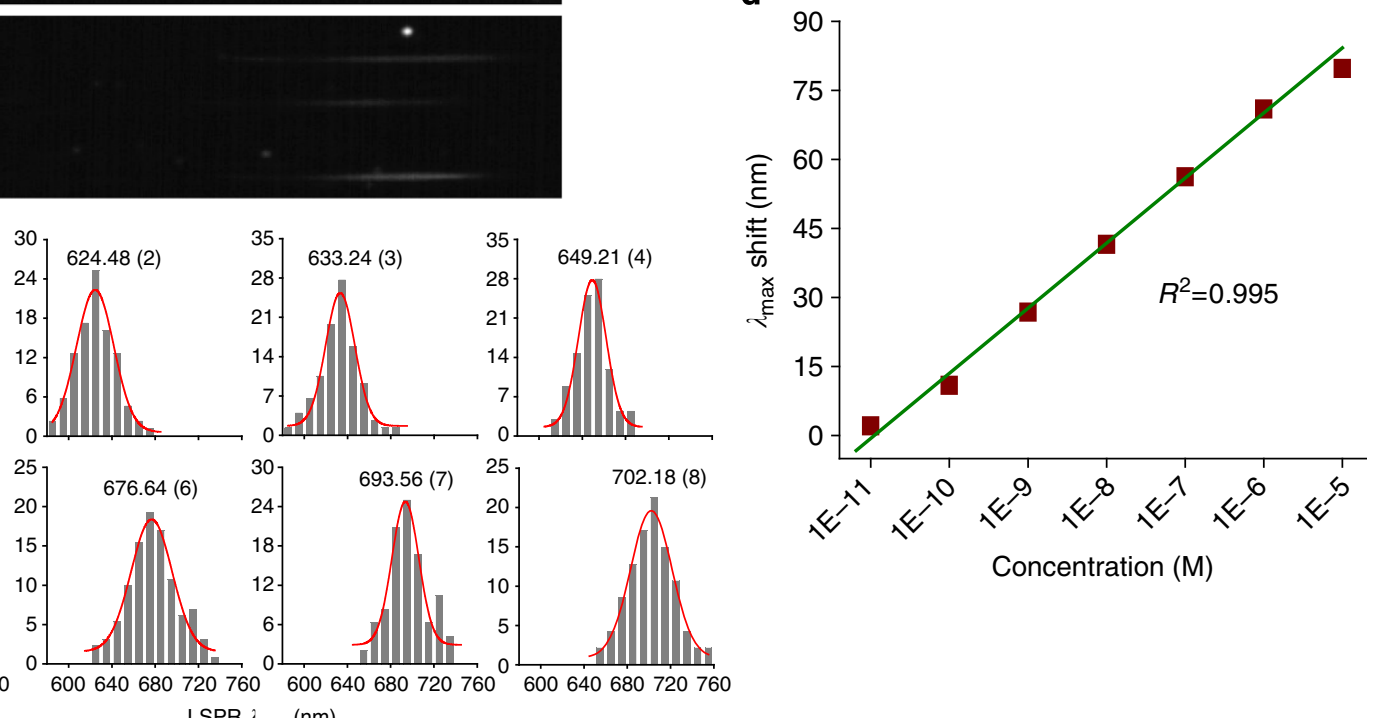

LSPR $\lambda_{\max }(\mathrm{nm})$

Figure 2 | Ultrasensitive sulphide sensing with single-particle dark-field spectral imaging. Typical single-particle spectral images (a) before and (b) after adding $\mathrm{Na}_{2} \mathrm{~S}$; a redshift is depicted as the displacement of the first-order streak towards the right. Scale bar, $2 \mu \mathrm{m}$. (c) Distribution of LSPR peak wavelengths after adding (1) $0 \mathrm{nM},(2) 0.01 \mathrm{nM},(3) 0.1 \mathrm{nM},(4) 1 \mathrm{nM},(5) 10 \mathrm{nM}$, (6) $100 \mathrm{nM}$, (7) $1 \mu \mathrm{M}$ and (8) $10 \mu \mathrm{M} \mathrm{Na} \mathrm{N}_{2} \mathrm{~S}$. Over 100 particles were counted in each case. (d) The centre of the $\lambda_{\max }$ distribution as a function of $\mathrm{Na}_{2} \mathrm{~S}$ concentration added.

$C_{\mathrm{S} \text {,eff. }}$ When $C_{\mathrm{S}, 0}$ is very small, there is a large fraction of unreacted Ag, the silver shell would act like a sponge and accumulate sulphide extensively, making $C_{\mathrm{S}, \text { eff }}$ larger than $C_{\mathrm{S}, 0}$. When $C_{\mathrm{S}, 0}$ is large, a substantial amount of $\mathrm{Ag}_{2} \mathrm{~S}$ forms quickly to cover the entire silver shell surface and hinder $\mathrm{Na}_{2} \mathrm{~S}$ in the solution from reacting with $\mathrm{Ag}$ atoms buried under the $\mathrm{Ag}_{2} \mathrm{~S}$ cover, so the apparent $C_{\mathrm{S} \text {,eff }}$ could be much smaller than $C_{\mathrm{S}, 0}$. It is important to note that to maintain the dynamic balance of the chemical potential of the sulphides reachable to the nanoprobe, $C_{S \text {, eff }}$ must be equal to the sum of the concentration of sulphide in the form of $\mathrm{Ag}_{2} \mathrm{~S}, C_{\mathrm{Ag}_{2} \mathrm{~S}}=C_{\mathrm{S}, \mathrm{NP}}$, and the concentration of free sulphide accessible to the $\mathrm{Ag}$ atoms. A reasonable approximation for such a kinetic equilibrium is a Freundlich-absorption relationship, $C_{\mathrm{S} \text {,eff }}=K C_{\mathrm{S}, 0}{ }^{(1 / p)}$, where $K$ and $p$ are both system-related constants ${ }^{47}$. The $p$-value would be $>1.0$ when $C_{S, 0}$ is small and be $<1.0$ when $C_{S, 0}$ is very large. On the other hand, from the viewpoint of reaction kinetics, $\mathrm{Ag}_{2} \mathrm{~S}$ formation on the $\mathrm{PNP}$ surface can be described by a pseudo first-order reaction with $\mathrm{O}_{2}$ in excess (in biological systems, the concentration of dissolved $\mathrm{O}_{2}$ is generally in the sub-mM to $\mu \mathrm{M}$ range, which is much larger than the effective concentration of sulphide $C_{\mathrm{S} \text {,eff }}$ in the tens of $\mathrm{nM}$ range that actually participated in the reaction)

$$
\frac{\mathrm{d} C_{\mathrm{Ag}_{2} \mathrm{~S}}}{d \mathrm{t}}=k_{\mathrm{obs}} \cdot\left(C_{\mathrm{S}, \text { eff }}-C_{\mathrm{Ag}_{2} \mathrm{~S}}\right)
$$

where $C_{\mathrm{S}, \text { eff }}-C_{\mathrm{Ag}_{2} \mathrm{~S}}$ is the effective concentration of free sulphide, and $k_{\mathrm{obs}}$ is the observed reaction rate. Just as $C_{\mathrm{S} \text {,eff }}-C_{\mathrm{Ag}_{2} \mathrm{~S}}$ is a corrected form of the $\mathrm{Na}_{2} \mathrm{~S}$ concentration applied, $k_{\text {obs }}$ should be the corrected form of the actual reaction rate constant, $k$, for the silver-sulphide reaction. It is because the silver shell contains multiple layers of silver atoms. Increasing the $\mathrm{Ag}_{2} \mathrm{~S}$ fraction not only reduces the availability and accessibility of free Ag atoms but also makes the collisions and reactions between silver atoms and sulphide ions more difficult and less efficient. Thus, $k_{\mathrm{obs}}$ can be corrected to $\left\{\left(A-a \cdot C_{\mathrm{Ag}_{2} \mathrm{~S}}\right) / A\right\} \cdot k$ (ref. 48), where $A$ is a preexponential Arrhenius factor, and $a$ is the site hindrance factor related to $\mathrm{Ag}_{2} \mathrm{~S}$ generated on the PNP surface. The solution to the above equations is (Supplementary Note 2):

$$
\frac{C_{\mathrm{Ag}, \mathrm{o}}}{C_{\mathrm{Ag}, \mathrm{o}}-2 C_{\mathrm{S}, \mathrm{eff}}} \ln \left(\frac{C_{\mathrm{Ag}, \mathrm{o}}-2 C_{\mathrm{Ag}_{2} \mathrm{~s}}}{C_{\mathrm{Ag}, \mathrm{o}}} \cdot \frac{C_{\mathrm{S}, \mathrm{eff}}}{C_{\mathrm{S}, \mathrm{eff}}-C_{\mathrm{Ag}_{2} \mathrm{~s}}}\right)=k t
$$

With $C_{\mathrm{Ag}, 0}$ and $k$ as constants and $C_{\mathrm{S} \text {,eff }}$ assuming to be a constant (for simplicity), $C_{\mathrm{Ag} 2 \mathrm{~S}}, C_{\mathrm{S}, \mathrm{NP}}$ and $\Delta \lambda_{\max }$ as a function of time can be calculated on the basis of equation 2 to either fit or be compared with the experimental data obtained when specific $\mathrm{Na}_{2} \mathrm{~S}$ concentrations are applied.

Figure $3 a-d$ show both measured and calculated timedependent spectral shifts of four single AuNR-Ag NPs when they were exposed to constant concentrations of $1 \mathrm{nM}, 10 \mathrm{nM}$, $100 \mathrm{nM}$ and $1 \mu \mathrm{M} \mathrm{Na} \mathrm{Na}_{2} \mathrm{~S}$, respectively. The experimental results show that in all cases, $\Delta \lambda_{\max }$ increase rapidly initially and then slow down. While the applied $\mathrm{Na}_{2} \mathrm{~S}$ concentrations differ by as much as 1,000 times, the difference in $\Delta \lambda_{\max }$, even at the longest time point recorded ( $t=20 \mathrm{~min})$, is less than 10 times. To fit these measured results with the above equations, the $p$-value is adjusted individually to obtain the closest fit for each $\mathrm{Na}_{2} \mathrm{~S}$ concentration. From the $P$-values obtained, we determined $C_{\mathrm{S} \text {, eff }}$ to be $4.2 \mathrm{nM}$, $12 \mathrm{nM}, 17 \mathrm{nM}$ and $25 \mathrm{nM}$ when the bulk $\mathrm{Na}_{2} \mathrm{~S}$ concentrations are $1 \mathrm{nM}, 10 \mathrm{nM}, 100 \mathrm{nM}$ and $1 \mu \mathrm{M}$, respectively. As expected, there is a sulphide accumulation effect at low $\mathrm{Na}_{2} \mathrm{~S}$ concentrations $(1 \mathrm{nM})$ and a sulphide hindrance effect at high $\mathrm{Na}_{2} \mathrm{~S}$ concentrations $(100 \mathrm{nM}$ and $1 \mu \mathrm{M})$. Notice that because we cannot measure the exact effective volume or the 'reachable range' of a PNP probe, the values of $C_{\mathrm{Ag}, 0}, k, p$ as well as $C_{\mathrm{S}, \mathrm{eff}}$, or 

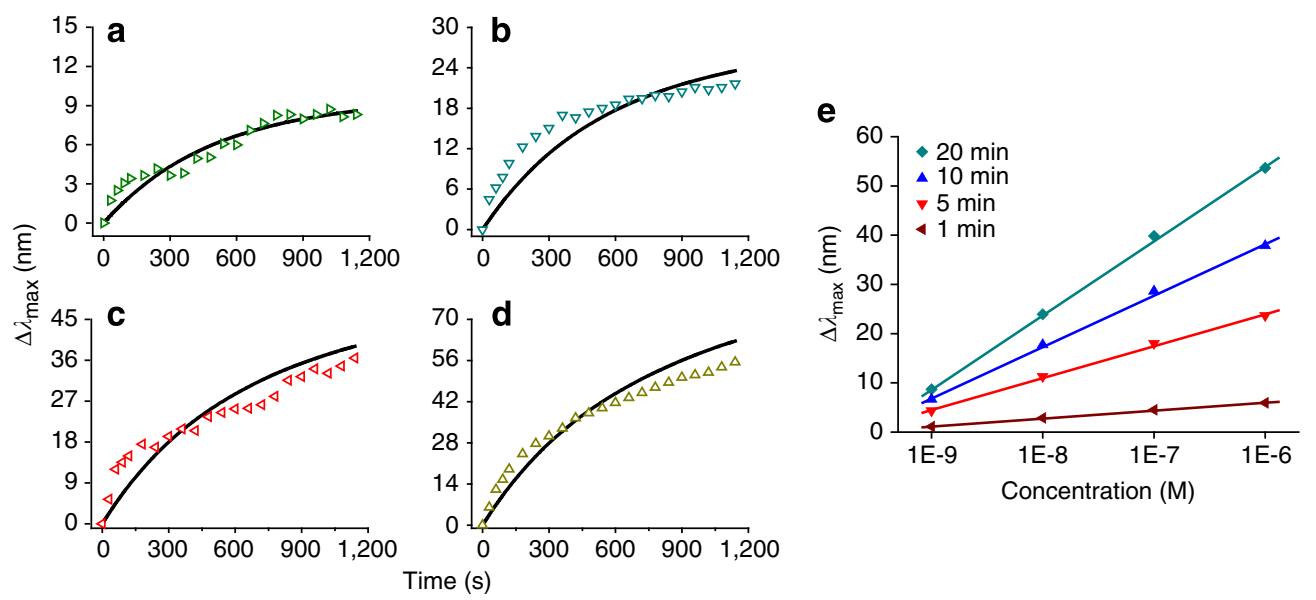

Figure 3 | PNP spectral shift rate varies as a function of sulphide concentration. Measured (hollow triangles) and calculated (black lines) timedependent spectral shifts of four single AuNR-Ag nanoprobes exposed to constant concentrations of $1 \mathrm{nM}(\mathbf{a}), 10 \mathrm{nM}(\mathbf{b}), 100 \mathrm{nM}(\mathbf{c})$ and $1 \mu \mathrm{M}(\mathbf{d}) \mathrm{Na} 2 \mathrm{~S}$. (e) Calculated $\Delta \lambda_{\max }$ values at various time points as a function of applied $\mathrm{Na}_{2} \mathrm{~S}$ concentration.

whether the particle accumulates or hinders $\mathrm{Ag}_{2} \mathrm{~S}$ formation, are only relative. In other words, $C_{\mathrm{S}, \text { eff }}$ is only conceptually significant in the present study, and more rigorous simulations are required to reveal whether this entity is quantitatively valid. Nevertheless, the fits to the experiments at all $\mathrm{Na}_{2} \mathrm{~S}$ concentrations and the trends of the corresponding $C_{\mathrm{S} \text {,eff }}$ values are reliable, as they are bound by actual measurements. Importantly, when we plot the calculated $\Delta \lambda_{\max }$ values versus the logarithm of applied $\mathrm{Na}_{2} \mathrm{~S}$ concentration at every time point, a good linear dependence is always obtained (Fig. 3e). This is consistent with the experimental results shown in Figure $2 c$, further demonstrating that our theoretical model is a rational explanation of the experimental observations.

One consequential implication here is that through dynamic equilibrium between $C_{\mathrm{S}, 0}$ and $C_{\mathrm{S}, \text { eff }}$ or the Freundlich-absorption relationship, the sulphide PNP probes effectively 'project' external sulphide concentrations that span widely over several orders of magnitude to a narrow concentration range of surface-bound sulphide (for example, $0-30 \mathrm{nM}$, as shown in Supplementary Fig. S4) that matches the detectable range of the probe's spectral response, although at the cost of reduced sensitivity and delayed responses to concentration changes. If such a dynamic equilibrium is established quickly, a reasonable assumption given that the nanoprobe is very small and $\mathrm{Ag}_{2} \mathrm{~S}$ formation is very fast, we can indirectly obtain local sulphide concentrations in real time by using time-dependent $\Delta \lambda_{\max }$ plots of single PNPs under various fixed sulphide concentrations as external calibration curves (Fig. 4a).

Notice that while the $\mathrm{Ag}_{2} \mathrm{~S}$ formation itself is irreversible, it is the observed reaction rate on the PNP surface that is responsive to variations in sulphide concentrations in the surrounding environment. As long as free silver atoms are available, the observed $\mathrm{Ag}_{2} \mathrm{~S}$ formation rate as well as the $\lambda_{\max }$ shifting rate would increase as the external sulphide concentration increases and vice versa. Figure $4 \mathrm{~b}, \mathrm{c}$ show $\Delta \lambda_{\max }$ values simulated using the equations derived earlier and the calculated sulphide concentrations using Fig. $3 \mathrm{e}$ as the calibration curves, respectively, as a function of time upon input of a square-pulse of external sulphide. The trend observed for the recovered timedependent variation in sulphide concentrations is very similar to that of the input, although there is some delay during the concentration reduction process because of the integrative nature of the nanoprobe. The slight deviations in the recovered sulphide concentration values are most likely due to the oversimplification of our derivations and the less accurate measurement(s) of analytical working curves, which are systematic factors that can likely be corrected with the future development of more rigorous theories and better experimental conditions. Figure $4 \mathrm{~d}$ shows the spectral shifts measured after alternately applying $5 \mu \mathrm{M} \mathrm{Na}_{2} \mathrm{~S}$ and pure water to an AuNR-Ag particle immobilized in a flow channel. The calculated local sulphide concentration and its variation are satisfactory (Fig. 4e). Therefore, this PNP nanoprobe is capable of tracking sulphide concentration fluctuations.

Real-time $\mathrm{H}_{2} \mathrm{~S}$ mapping in live cells. To determine whether these single AuNR-Ag PNPs and this indirect method for sulphide analysis is applicable to live cells, we incubated the PNPs with HeLa cells. Standard assays indicate that these PNPs do not aggregate in cell culture media with serum and have almost no cytotoxicity (data not shown). After incubation for $4 \mathrm{~h}$, some PNPs were found in the cytoplasm of the cells, where the PNPs appeared as bright orange spots under dark-field illumination. Because the PNPs have much higher intensities than intracellular organelles, the zero-order spots (position) and the first-order streaks (spectrum) of the nanoprobes inside the cell can be readily obtained, especially after background subtraction (Supplementary Fig. S5). As a control experiment, we monitored the spectra of individual PNPs continuously for $40 \mathrm{~min}$ in the absence of sulphide. The variation in their $\lambda_{\max }$ values was found to be $\sim 2 \mathrm{~nm}$ (Supplementary Fig. S6), indicating that these PNPs are highly stable spectrally within the intracellular environment.

Next, $\mathrm{Na}_{2} \mathrm{~S}$ was added to the cell culture medium to a final concentration of $0.1 \mu \mathrm{M}$. Time-dependent colour images and spectral variations of two single PNPs were recorded simultaneously for $40 \mathrm{~min}$. During this time period, as shown in Figure 5 and Supplementary Fig. S7, the $\lambda_{\max }$ of one particle (red circle, P1) redshifted by $\sim 40 \mathrm{~nm}$, while the other (green circle, P2) changed $\sim 20 \mathrm{~nm}$. Although the two AuNR-Ag particles were not exactly the same in terms of their aspect ratios (their initial $\lambda_{\max }$ difference was $\sim 20 \mathrm{~nm}$ ), single-particle heterogeneities alone cannot account for such a large spectral response difference according to our simulations (Supplementary Fig. S8). Therefore, the local sulphide concentration and its accumulations around the two particles must be different. Comparing the fitted $\Delta \lambda_{\max }$ plots of P1 and P2 with $\Delta \lambda_{\max }$ reference plots in Fig. 3, the sulphide concentrations experienced by P1 apparently falls between $10 \mathrm{nM}$ to $100 \mathrm{nM}$, while that experienced by P2 falls between $1 \mathrm{nM}$ and $10 \mathrm{nM}$ (Fig. 5d). Using the curves in Fig. 3e as 

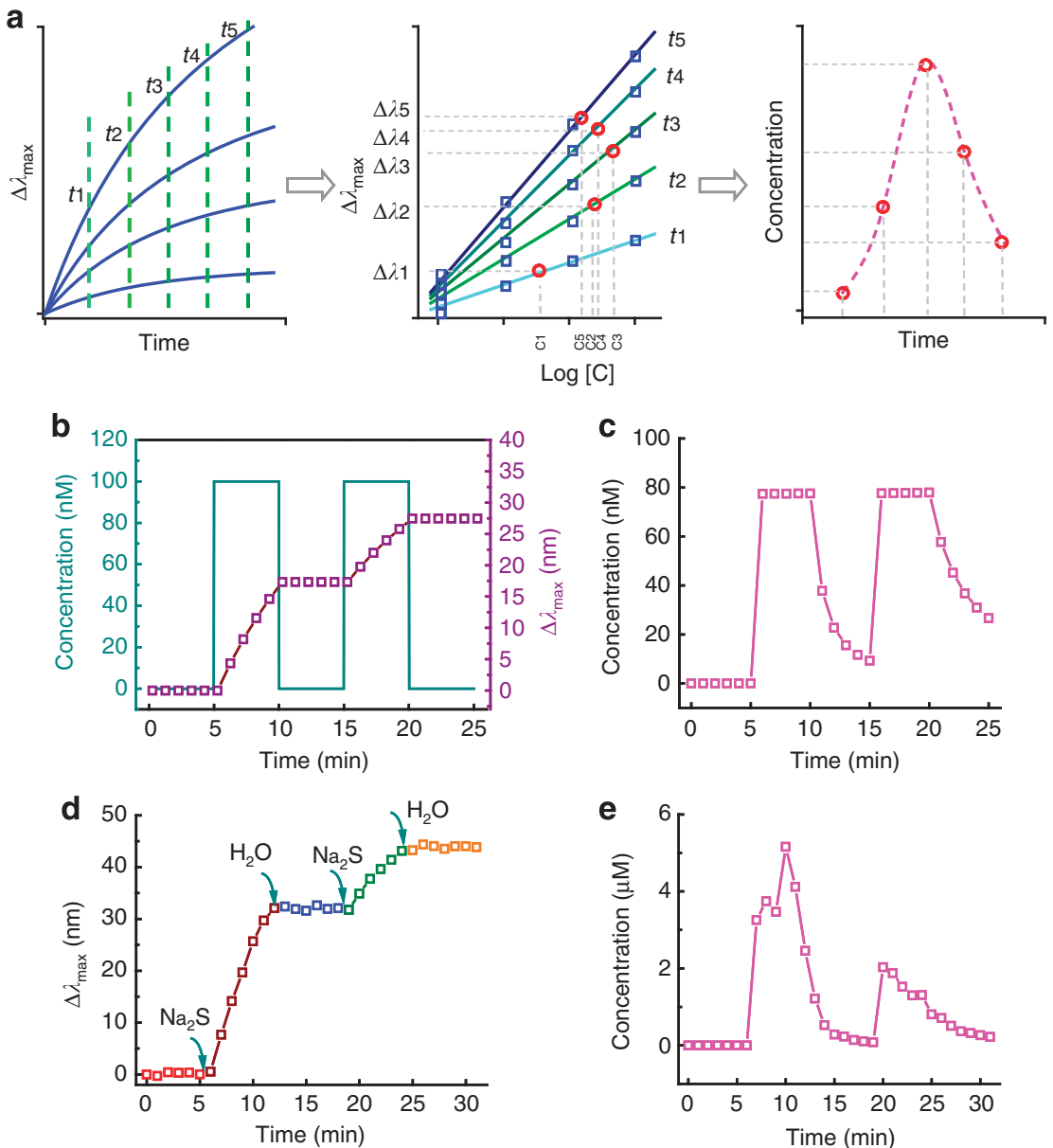

Figure 4 | Sulphide concentration fluctuations can be determined from PNP spectral shift rates. (a) Schematic illustration of the 'integration sensing' concept pertaining to the AuNR-Ag nanoprobes. Local sulphide concentrations can be obtained indirectly by using time-dependent $\Delta \lambda_{\text {max }}$ (increase) plots at various constant sulphide concentrations as external calibration curves. $(\mathbf{b}, \mathbf{c})$ The simulated $\lambda_{\max }$ shift and the recovered sulphide concentration as a function of time upon a square-pulse input of $100 \mathrm{nM} \mathrm{Na}_{2} \mathrm{~S}$. (d,e) The experimentally measured $\lambda_{\max }$ shift and the calculated sulphide concentration as a function of time after alternately adding $40 \mu \mathrm{l}$ of $5 \mu \mathrm{M} \mathrm{Na}{ }_{2} \mathrm{~S}$ solution and pure water.

external calibration curves and the $\Delta \lambda_{\max }$ values from data interpolations in Fig. $5 \mathrm{~d}$ as inputs, the time-dependent local sulphide concentration increases are determined for both particles (Fig. 5e). From $2 \mathrm{~min}$ to $40 \mathrm{~min}$, the apparent local concentration of sulphide experienced by $\mathrm{P} 1$ changes from $5.8 \mathrm{nM}$ to $41.8 \mathrm{nM}$, whereas that experienced by $\mathrm{P} 2$ changes from $0.5 \mathrm{nM}$ to $3.8 \mathrm{nM}$. These values are all smaller than the $100 \mathrm{nM}$ sulphide concentration supplied externally but are reasonable for such an 'integration sensor'. Because sulphide is highly diffusive and reactive, the large difference between $\mathrm{P} 1$ and $\mathrm{P} 2$ is most likely an indication of heterogeneous diffusion, distribution or consumption of sulphide throughout the cell. Although the indirectly measured sulphide concentrations may deviate more or less from the true values due to some systematic errors, the magnitudes as well as the trends of the calculated values are nevertheless reliable. Most previous reports regarding fluorescent sulphide probes were only able to provide static whole-cell images and reveal qualitative fluorescence intensity differences (among these images) after 1530 min external stimulations with various sulphide concentrations in the $\mu \mathrm{M}$ range $24,26,28,29$ To our knowledge, ours is not only the first demonstration of live cell sulphide imaging with $\mathrm{nM}$ sensitivity but is also the first time that local variations in intracellular sulphide levels have been tracked dynamically.

In addition to sensing sulphide supplied from outside the cell, we also determined if our PNP probes could be utilized to map spatially resolved concentrations of sulphides generated inside live cells. Previous studies have demonstrated that the cystathionine $\beta$-synthase (CBS) enzyme, which is involved in $\mathrm{H}_{2} \mathrm{~S}$ generation using cysteine and homocysteine as sulphide sources, is strongly expressed in nonconfluent HepG2 cells, but the activity of CBS is significantly decreased after the cells are treated with human insulin ${ }^{49-52}$. Thus, we adopted normal and insulin-pretreated HepG2 cells as model cells, incubated them with the PNPs, and examined whether variations in intracellular $\mathrm{H}_{2} \mathrm{~S}$ production could be detected. As seen in Figure 6 and Supplementary Fig. S9, after adding cysteine and homocysteine to the cell culture medium for $30 \mathrm{~min}$, the $\lambda_{\max }$ shifts of two representative PNPs in the normal HepG2 cell were significantly larger than those in the cell pretreated with human insulin. The calculated intracellular $\mathrm{H}_{2} \mathrm{~S}$ concentration around the PNPs in the normal HepG2 cells rose quickly to $\sim 100 \mathrm{nM}$ and then declined gradually (Fig. 6b), most likely because of an increase in the gaseous-transmitter-induced sulphide catabolism or the diffusion of sulphide into the extracellular medium. In contrast, the $\mathrm{H}_{2} \mathrm{~S}$ concentration in the insulin-pretreated HepG2 cell increased slowly to only $\sim 1 \mathrm{nM}$ (Fig. $6 \mathrm{~d}$ ), consistent with the prediction that the CBS activity as well as $\mathrm{H}_{2} \mathrm{~S}$ production was greatly reduced. In the negative control experiment, no PNP spectral shifts were observed if cysteine and homocysteine were not added (Supplementary Fig. S9e). Hence, differences in endogenous $\mathrm{H}_{2} \mathrm{~S}$ 

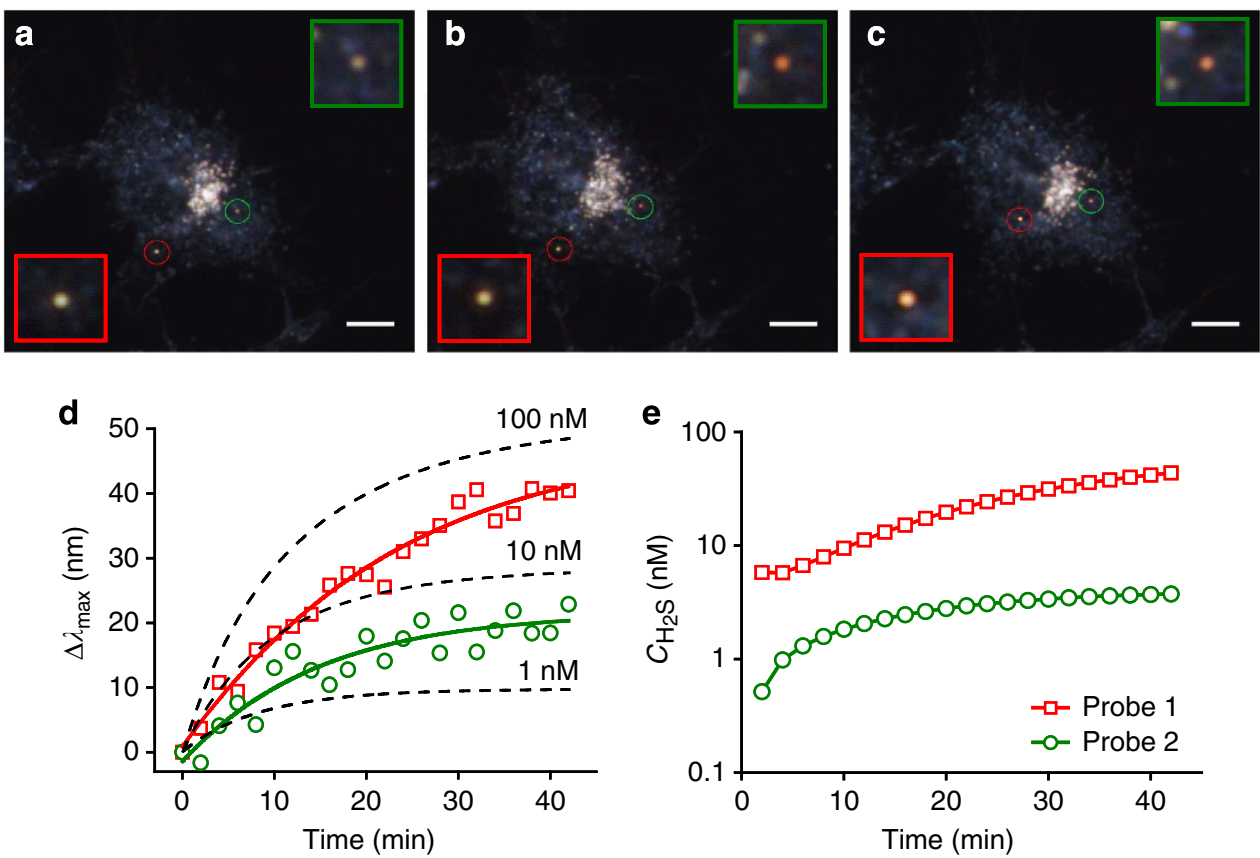

Figure 5 | Local variations of intracellular sulphide levels can be determined in real time. (a-c) Representative images showing the gradual colour changes of two individual PNPs after adding $0.1 \mu \mathrm{M} \mathrm{Na}_{2} \mathrm{~S}$ to the cell culture medium for (a) $2 \mathrm{~min}$, (b) $26 \mathrm{~min}$ and (c) $42 \mathrm{~min}$. Scale bar, $10 \mu \mathrm{m}$. The red and green square inserts are enlarged images of the two circled PNPs. (d) Observed (hollow dots) and fitted (lines) time-dependent $\lambda_{\max }$ shifts of the two particles. The calculated dynamic $\Delta \lambda_{\max }$ shifts at 1, 10 and $100 \mathrm{nM}$ adapted from Fig. 3a-d are also plotted (dashed lines) for reference. (e) Calculated time-dependent change in local sulphide concentrations surrounding the two particles according to the fitted results in d, using Fig. $3 e$ as the external calibration curves.
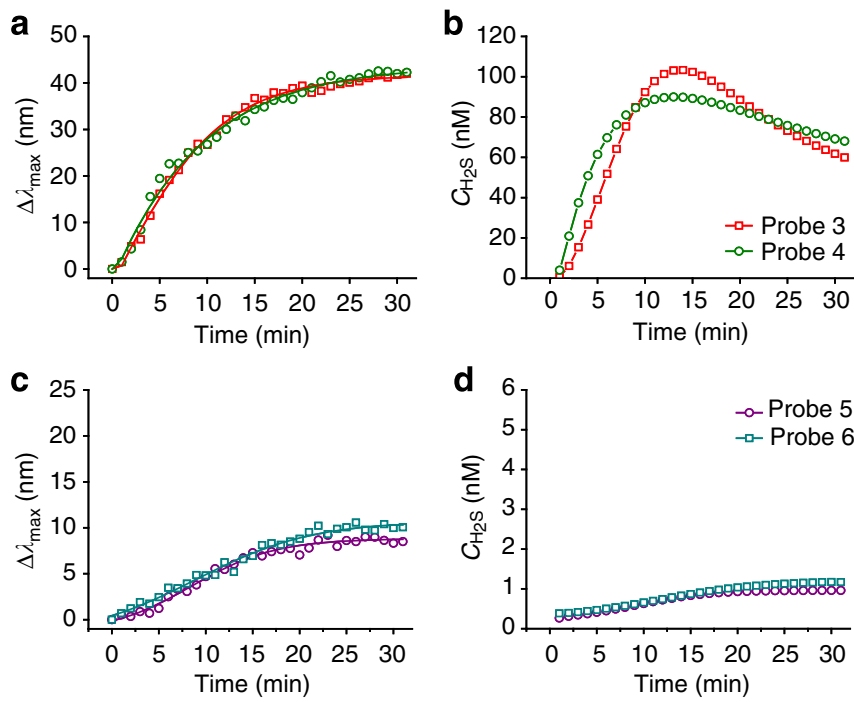

Figure 6 | Endogenous sulphide concentrations can be determined. Observed (hollow dots) and fitted (lines) time courses of $\Delta \lambda_{\max }$ shifts of representative PNPs in HepG2 cells without (a) and with (c) insulin pretreatment. Cysteine and homocysteine were used as the $\mathrm{H}_{2} \mathrm{~S}$ source. Calculated real-time local $\mathrm{H}_{2} \mathrm{~S}$ concentration variations for these two cases are shown in $\mathbf{b}$ and $\mathbf{d}$, respectively.

levels were successfully tracked in real time, and our AuNR-Ag core-shell PNPs can be utilized to map spatial-temporal distributions of biologically produced $\mathrm{H}_{2} \mathrm{~S}$ inside live cells.

\section{Discussion}

This study introduces a new method for highly sensitive sulphide mapping on the basis of single-particle spectral imaging using
AuNR-Ag core-shell nanoparticles as probes. The integrative$\mathrm{Ag}_{2} \mathrm{~S}$-formation-induced time-dependent spectral shifts of the nanoprobes are not only highly selective towards sulphide but also show a linear logarithmic dependence on sulphide concentrations at all time points. A theoretical model was established that successfully explains the experimental observations and suggests that local sulphide concentrations can be determined from kinetic measurements of spectral shifts of the nanoprobes. This model allows real-time detection of local variations of sulphide levels in live cells with $\mathrm{nM}$ sensitivity over a large dynamic range. Being a technique that essentially measures 'localized concentrations' at the single-molecule level, this integrative sensing approach, based on single PNP imaging platforms and probes, could potentially be utilized to develop highly sensitive detection methods for other small molecules or ions.

\section{Methods}

Chemicals and PNP preparation. Human insulin, pyridoxal 5-phosphate and polyvinyl pyrrolidone ( $M W=29,000)$ were obtained from Sigma-Aldrich. All other reagents were AR grade and were purchased from Shanghai Sinopharm. AuNR synthesis and purification procedures were adopted elsewhere ${ }^{53,54}$. The resulting AuNRs were used as seeds for the growth of AuNR-Ag NPs according to the literature with some modifications ${ }^{55}$. In brief, $0.8 \mathrm{ml}$ of purified AuNR solution was mixed with $2.4 \mathrm{ml}$ of $3 \%$ polyvinyl pyrrolidone aqueous solution, after which freshly prepared ammoniacal silver nitrate $(120 \mu \mathrm{l}, 1 \mathrm{mM})$ was added to the mixture while stirring, followed by addition of ascorbic acid solution $(120 \mu \mathrm{l}$, $10 \mathrm{mM}$ ). Finally, the solution was placed into a water bath and kept undisturbed at $30^{\circ} \mathrm{C}$ until the colour change was complete. The as-prepared PNP probes were characterized with ultraviolet-vis spectroscopy (Shimadzu UV-1800, Japan) and transmission electron microscopy (TEM, JEM 1230, JEOL, Japan).

Single-particle dark-field spectral imaging. The experiments employed a singleparticle spectral dark-field microscope with a wavelength resolution of $1.73 \mathrm{~nm}$ per pixel (after calibration). The set-up was composed of a Nikon 80i optical microscope (Japan), an oil-immersion dark-field condenser (numerical aperture 1.201.43), a $60 \times$ Plan Fluor objective, a TGBS (70 grooves per mm, Edmund Optics), a CoolSNAP HQ2 CCD camera (with an optimal spectral response window of 
$470-630 \mathrm{~nm}$ with quantum efficiency $>60 \%$, Roper Scientific) and an Olympus DP72 colour CCD camera.

In vitro sulphide detection. To detect sulphide with dark-field spectral microscopy, home-built flow channels were prepared with coverslips and double-sided adhesive tape ( $5 \mathrm{~mm}$ wide, $\sim 40 \mu \mathrm{l}$ total volume). Before each experiment, $20 \mu \mathrm{l}$ of AuNR-Ag PNPs $(\sim 25 \mathrm{pM})$ was injected into a flow channel, and the PNPs were absorbed onto the glass surface by adjusting the $\mathrm{pH}$ of the solution. After $20 \mathrm{~min}$, unabsorbed PNPs were washed away by rinsing with water three times. Then, $40 \mu \mathrm{l}$ $\mathrm{Na}_{2} \mathrm{~S}$ solution was injected into the channel and LSPR scattering spectral images of individual nanoparticles were recorded using the single-particle spectral microscope at different time points with an exposure time of $1 \mathrm{~s}$. This process was repeated for $\mathrm{Na}_{2} \mathrm{~S}$ solutions of various concentrations. Image analyses were performed using the ImageJ software, and LSPR spectral maxima of each nanoparticle were obtained using the peakfit code in the Matlab program.

Intracellular sulphide mapping. Human cervical cancer (HeLa cell) lines and human hepatoma (HepG2) cells were obtained from the American Type Culture Collection (ATCC, USA). The cells were maintained in Dulbecco's modified Eagle's medium (Gibco) supplemented with $10 \%$ fetal bovine serum (Gibco) at $37^{\circ} \mathrm{C}, 5 \%$ $\mathrm{CO}_{2}$ in a humidified atmosphere. The cells were cultured on a cleaned cover-glass in a plastic cell culture dish. Internalization of nanoprobes was realized by adding $5 \mu \mathrm{l}(50 \mathrm{pM})$ of AuNR-Ag PNPs to the cell culture dish and incubating for $4 \mathrm{~h}$. To study sulphide transport into the cytoplasm and to monitor intracellular sulphide in real time, the cover-glass was inverted and placed on a glass slide with a concave cavity in the centre, to which the cell culture medium containing $100 \mathrm{nM} \mathrm{Na}_{2} \mathrm{~S}$ was applied. Because the redshifting rate of the PNP spectra in live cells was slow, the LSPR scattering images were recorded every 2 min with an exposure time of $2 \mathrm{~s}$.

To suppress CBS activity in HepG2 cells, human insulin was added (with a final concentration of $1 \mu \mathrm{M}$ ) to the cell culture medium, and the cells were incubated for $48 \mathrm{~h}$. For endogenous $\mathrm{H}_{2} \mathrm{~S}$ imaging in live cells, normal and insulin-pretreated HepG2 cells were first incubated with the PNPs for $4 \mathrm{~h}$. The CBS cofactor (pyridoxal 5-phosphate, at a final concentration of $200 \mu \mathrm{M}$ ) and the sulphide source ( $1 \mathrm{mM}$ cysteine and $1 \mathrm{mM}$ homocysteine) were then added to the culture medium, and the LSPR spectra of PNPs in the HepG2 cells with or without human insulin pretreatment were monitored using single-particle dark-field spectral microscopy. To track endogenous $\mathrm{H}_{2} \mathrm{~S}$ levels, LSPR spectral images were recorded every minute with an exposure time of $2 \mathrm{~s}$.

\section{References}

1. Coletta, C. et al. Hydrogen sulfide and nitric oxide are mutually dependent in the regulation of angiogenesis and endothelium-dependent vasorelaxation. Proc. Natl Acad. Sci USA 109, 9161-9166 (2012).

2. Szabo, C. Hydrogen sulphide and its therapeutic potential. Nat. Rev. Drug Discov. 6, 917-935 (2007).

3. Łowicka, E. \& Bettowski, J. Hydrogen sulfide (H2S) - the third gas of interest for pharmacologists. Pharmacol. Rep. 59, 4-24 (2007).

4. Wang, R. Two's company, three's a crowd: can $\mathrm{H} 2 \mathrm{~S}$ be the third endogenous gaseous transmitter? FASEB J. 16, 1792-1798 (2002).

5. Yang, G. et al. $\mathrm{H} 2 \mathrm{~S}$ as a physiologic vasorelaxant: hypertension in mice with deletion of cystathionine $\gamma$-lyase. Science 322, 587-590 (2008).

6. Papapetropoulos, A. et al. Hydrogen sulfide is an endogenous stimulator of angiogenesis. Proc. Natl Acad. Sci. USA 106, 21972-21977 (2009).

7. Leffler, C. W., Parfenova, H., Jaggar, J. H. \& Wang, R. Carbon monoxide and hydrogen sulfide: gaseous messengers in cerebrovascular circulation. J. Appl. Physiol. 100, 1065-1076 (2006).

8. Kimura, H. Hydrogen sulfide as a neuromodulator. Mol. Neurobiol. 26, 13-19 (2002).

9. Abe, K. \& Kimura, H. The possible role of hydrogen sulfide as an endogenous neuromodulator. J. Neurosc. 16, 1066-1071 (1996).

10. Li, L. et al. Hydrogen sulfide is a novel mediator of lipopolysaccharide-induced inflammation in the mouse. FASEB J. 19, 1196-1198 (2005).

11. Yang, G., Sun, X. \& Wang, R. Hydrogen sulfide-induced apoptosis of human aorta smooth muscle cells via the activation of mitogen-activated protein kinases and caspase-3. FASEB J. 18, 1782-1784 (2004).

12. Kaneko, Y., Kimura, Y., Kimura, H. \& Niki, I. L-Cysteine inhibits insulin release from the pancreatic $\beta$-cell. Diabetes 55, 1391-1397 (2006).

13. Peng, Y.-J. et al. $\mathrm{H}_{2} \mathrm{~S}$ mediates $\mathrm{O}_{2}$ sensing in the carotid body. Proc. Natl Acad. Sci. USA 107, 10719-10724 (2010).

14. Elrod, J. W. et al. Hydrogen sulfide attenuates myocardial ischemia-reperfusion injury by preservation of mitochondrial function. Proc. Natl Acad. Sci. USA 104, 15560-15565 (2007).

15. Kamoun, P., Belardinelli, M. C.,, Chabli, A., Lallouchi, K. \& Chadefaux, V. B., Endogenous hydrogen sulfide overproduction in Down syndrome. Am. J. Med. Genet. A 116A, 310-311 (2003).
16. McGeer, E. G. \& McGeer, P. L. Neuroinflammation in Alzheimer's disease and mild cognitive impairment: a field in its infancy. J. Alzheimers Dis. 19, 355-361 (2010).

17. Suzuki, K. et al. Hydrogen sulfide replacement therapy protects the vascular endothelium in hyperglycemia by preserving mitochondrial function. Proc. Natl Acad. Sci. USA 108, 13829-13834 (2011).

18. Jha, S., Calvert, J. W., Duranski, M. R., Ramachandran, A. \& Lefer, D. J. Hydrogen sulfide attenuates hepatic ischemia-reperfusion injury: role of antioxidant and antiapoptotic signaling. Am. J. Physiol. Heart Circ. Physiol. 295, H801-H806 (2008).

19. Jiménez, D. et al. A new chromo-chemodosimeter selective for sulfide anion. J. Am. Chem. Soc. 125, 9000-9001 (2003).

20. Schiavon, G., Zotti, G., Toniolo, R. \& Bontempelli, G. Electrochemical detection of trace hydrogen sulfide in gaseous samples by porous silver electrodes supported on ion-exchange membranes (solid polymer electrolytes). Anal. Chem. 67, 318-323 (1995).

21. Li, H., Tian, Y., Deng, Z. \& Liang, Y. An in situ photoelectrochemical determination of hydrogen sulfide through generation of CdS nanoclusters onto $\mathrm{TiO} 2$ nanotubes. Analyst 137, 4605-4609 (2012).

22. Radford-Knoery, J. \& Cutter, G. A. Determination of carbonyl sulfide and hydrogen sulfide species in natural waters using specialized collection procedures and gas chromatography with flame photometric detection. Anal. Chem. 65, 976-982 (1993).

23. Xuan, W., Sheng, C., Cao, Y., He, W. \& Wang, W. Fluorescent probes for the detection of hydrogen sulfide in biological systems. Angew. Chem. Int. Ed. 51, 2282-2284 (2012).

24. Chen, S., Chen, Z.-j., Ren, W. \& Ai, H.-w Reaction-based genetically encoded fluorescent hydrogen sulfide sensors. J. Am. Chem. Soc. 134, 9589-9592 (2012).

25. Sasakura, K. et al. Development of a highly selective fluorescence probe for hydrogen sulfide. J. Am. Chem. Soc. 133, 18003-18005 (2011).

26. Qian, Y. et al. Selective fluorescent probes for live-cell monitoring of sulphide. Nat. Commun. 2, 495 (2011).

27. Peng, H. et al. A fluorescent probe for fast and quantitative detection of hydrogen sulfide in blood. Angew. Chem. Int. Ed. 50, 9672-9675 (2011).

28. Lippert, A. R., New, E. J. \& Chang, C. J. Reaction-based fluorescent probes for selective imaging of hydrogen sulfide in living cells. J. Am. Chem. Soc. 133, 10078-10080 (2011).

29. Yu, C., Li, X., Zeng, F., Zheng, F. \& Wu, S. Carbon-dot-based ratiometric fluorescent sensor for detecting hydrogen sulfide in aqueous media and inside live cells. Chem. Commun. 49, 403-405 (2013).

30. Vitvitsky, V., Kabil, O. \& Banerjee, R. High turnover rates for hydrogen sulfide allow for rapid regulation of its tissue concentrations. Antioxid. Redox Sign. 17, 22-31 (2012)

31. Stewart, M. E. et al. Nanostructured plasmonic sensors. Chem. Rev. 108, 494-521 (2008).

32. Anker, J. N. et al. Biosensing with plasmonic nanosensors. Nat. Mater. 7, 442-453 (2008).

33. McFarland, A. D. \& Van Duyne, R. P. Single silver nanoparticles as real-time optical sensors with zeptomole sensitivity. Nano Lett. 3, 1057-1062 (2003).

34. Jain, P. K., Lee, K. S., El-Sayed, I. H. \& El-Sayed, M. A. Calculated absorption and scattering properties of gold nanoparticles of different size, shape, and composition: applications in biological imaging and biomedicine. J. Phys. Chem. B 110, 7238-7248 (2006).

35. Jain, P. K., Huang, X., El-Sayed, I. H. \& El-Sayed, M. A. Noble metals on the nanoscale: optical and photothermal properties and some applications in imaging, sensing, biology, and medicine. Acc. Chem. Res. 41, 1578-1586 (2008).

36. Zhang, L. et al. Single gold nanoparticles as real-time optical probes for the detection of NADH-dependent intracellular metabolic enzymatic pathways. Angew. Chem. Int. Ed 123, 6921-6924 (2011).

37. Novo, C., Funston, A. M. \& Mulvaney, P. Direct observation of chemical reactions on single gold nanocrystals using surface plasmon spectroscopy. Nat. Nano 3, 598-602 (2008).

38. Lilienfeld, S. \& White, C. E. A study of the reaction between hydrogen sulfide and silver. J. Am. Chem. Soc. 52, 885-892 (1930).

39. Zeng, J. et al. Selective sulfuration at the corner sites of a silver nanocrystal and its use in stabilization of the shape. Nano Lett. 11, 3010-3015 (2011).

40. Johnson, P. B. \& Christy, R. W. Optical constants of the noble metals. Phys. Rev. B 6, 4370-4379 (1972).

41. Bennett, J. M., Stanford, J. L. \& Ashley, E. J. Optical constants of silver sulfide tarnish films. J. Opt. Soc. Am. 60, 224-231 (1970).

42. Miller, M. M. \& Lazarides, A. A. Sensitivity of metal nanoparticle surface plasmon resonance to the dielectric environment. J. Phys. Chem. B 109, 21556-21565 (2005).

43. Dickinson, D. A. \& Forman, H. J. Cellular glutathione and thiols metabolism. Biochem. Pharmacol. 64, 1019-1026 (2002).

44. Stipanuk, M. H., Dominy, J. E., Lee, J.-I. \& Coloso, R. M. Mammalian cysteine metabolism: new insights into regulation of cysteine metabolism. J. Nutr. 136, 1652S-1659S (2006). 
45. Cheng, J., Liu, Y., Cheng, X., He, Y. \& Yeung, E. S. Real time observation of chemical reactions of individual metal nanoparticles with high-throughput single molecule spectral microscopy. Anal. Chem. 82, 8744-8749 (2010).

46. Novo, C., Funston, A. M., Pastoriza-Santos, I., Liz-Marzan, L. M. \& Mulvaney, $\mathrm{P}$. Influence of the medium refractive index on the optical properties of single gold triangular prisms on a substrate. J. Phys. Chem. C 112, 3-7 (2007).

47. Hill, T. L. Statistical mechanics of multimolecular adsorption. IV. the statistical analog of the BET constant $\mathrm{a}_{1} \mathrm{~b}_{2} / \mathrm{b}_{1} \mathrm{a}_{2}$. hindered rotation of a symmetrical diatomic molecule near a surface. J. Chem. Phys. 16, 181-189 (1948).

48. Seo, D., Park, G. \& Song, H. Plasmonic monitoring of catalytic hydrogen generation by a single nanoparticle probe. J. Am. Chem. Soc. 134, 1221-1227 (2011).

49. Ratnam, S. et al. Hormonal regulation of cystathionine $\beta$-synthase expression in liver. J. Biol. Chem. 277, 42912-42918 (2002).

50. Zou, C.-G. \& Banerjee, R. Tumor necrosis factor- $\alpha$-induced targeted proteolysis of cystathionine $\beta$-synthase modulates redox homeostasis. J. Biol. Chem. 278, 16802-16808 (2003).

51. Prudova, A. et al. S-adenosylmethionine stabilizes cystathionine $\beta$-synthase and modulates redox capacity. Proc. Natl Acad. Sci. USA 103, 6489-6494 (2006).

52. Singh, S., Padovani, D., Leslie, R. A., Chiku, T. \& Banerjee, R. Relative contributions of cystathionine $\beta$-synthase and $\gamma$-cystathionase to $\mathrm{H}_{2} \mathrm{~S}$ biogenesis via alternative trans-sulfuration reactions. J. Biol. Chem. 284, 22457-22466 (2009).

53. Nikoobakht, B. \& El-Sayed, M. A. Preparation and growth mechanism of gold nanorods (NRs) using seed-mediated growth method. Chem. Mater. 15, 1957-1962 (2003).
54. Ni, W., Kou, X., Yang, Z. \& Wang, J. F. Tailoring longitudinal surface plasmon wavelengths, scattering and absorption cross sections of gold nanorods. ACS Nano 2, 677-686 (2008).

55. Becker, J. et al. Plasmonic focusing reduces ensemble linewidth of silver-coated gold nanorods. Nano Lett. 8, 1719-1723 (2008).

\section{Acknowledgements}

We are grateful for the financial support by NSFC 21127009, NSFC 91027037 and Hunan University 985 fund.

\section{Author contributions}

B.X., R.Z., Y.H. and E.S.Y. designed and performed the experiments, analysed the data and wrote the manuscript. J.H. and Y.J. helped in sample preparation.

\section{Additional information}

Supplementary Information accompanies this paper at http://www.nature.com/ naturecommunications

Competing financial interests: The authors declare no competing financial interests.

Reprints and permission information is available online at http://npg.nature.com/ reprintsandpermissions/

How to cite this article: Xiong, B. et al. Highly sensitive sulphide mapping in live cells by kinetic spectral analysis of single Au-Ag core-shell nanoparticles. Nat. Commun. 4:1708 doi: 10.1038/ncomms2722 (2013). 